\title{
Surgical Pleth Index and Analgesia Nociception Index for intraoperative analgesia in patients undergoing neurosurgical spinal procedures, a comparative study
}

\author{
Dostalova V, Schreiberova J, Dopitova P, Dostal P \\ Dept. of Anaesthesiology and Intensive Care Medicine, \\ Charles University, Faculty of Medicine Hradec Kralove, University Hospital Hradec Kralove, \\ Czech Republic
}

Background and Goal of the Study

The Surgical Pleth Index (SPI) and the Analgesia Nociception Index (ANI) have been recently suggested for the non-invasive intraoperative monitoring of nociception/anti-nociception balance ${ }^{1}$. The goal of this study was to compare intraoperative use of opioids guided either by ANI, SPI or anaesthesiologist's judgment during desflurane-sufentanil anaesthesia respectively.

\begin{abstract}
Material and Methods
72 adult ASA I - III patients scheduled for elective neurosurgical spinal procedures were randomized into the ANI group, SPI group and control group. Exclusion criteria included presence of non-sinus rhythm, pacemaker, planned postoperative ventilation, procedures with planned awake intervals, chronic pain with opioid medication, opioid addiction, epidural administration of local anaesthetic in combination with opioid, hormonal contraceptives. Anaesthesia and intraoperative use of opioids (sufentanil boluses based on body weight) were managed according to a strict protocol. The use of sufentanil was targeted to keep ANI value within the range of 50-70 in the ANI group and SPI value below individual post-induction baseline SPI value plus 10 points in the SPI group. In the control group, the use of opioids was left at anaesthesiologist's discretion. Results are expressed as mean $\pm \mathrm{SD}$ or as the median with interquartile ranges (IQR) based on the results of a test of normality of the distribution using a onesample Kolmogorov-Smirnov test. Intergroup differences were tested using ANOVA with a Student-Newman-Keuls test for all pair wise comparisons, a Kruskal Wallis test or a Chi-squared test, as appropriate.
\end{abstract}

\section{Results}

There were no differences in patient's baseline characteristics, duration and type of surgery, duration of anaesthesia, used concentrations of desflurane and total sufentanil dose (Table 1, Table 2).

Additional sufentanil boluses were administered earlier in the ANI and SPI groups in comparison with the control group ( $3^{\text {rd }}$ dose after $51.8 \pm$ 22.1 vs $52.7 \pm 14.8$ vs $84.5 \pm 24.8$ min respectively, $\mathrm{p}=0.001 ; 4^{\text {th }}$ dose after $61.3 \pm 30.1$ vs $57.2 \pm 14.1$ vs $120.0 \pm 26.2 \mathrm{~min}, \mathrm{p}=0.003$, and $5^{\text {th }}$ dose after $78.8 \pm 33.7$ vs $74.0 \pm 11.6$ vs $146.7 \pm 23.2$ min respectively, $\mathrm{p}=0.009)$, Figure 1 .

There were no differences in time to spontaneous breathing at the end of anaesthesia $(6.2 \pm 5.4$ vs $4.4 \pm 3.2$ vs $4.3 \pm 2.6$ min respectively, $\mathrm{p}=$ $0,207)$, postoperative pain scores, cortisol serum concentrations and complication rates between groups (Table 3 ).

\section{Discussion}

Our study had several limitations. Anaesthesia protocol could have influenced obtained results. Number of patients was limited and imbalances between groups, although statistically non-significant, could also have influenced obtained data.

\section{Conclusion}

Within limitations of this study, our findings suggest that both SPI and ANI monitoring provide clinically usable intraoperative sufentanil use guidance and significantly modify timing of sufentanil boluses in comparison with anaesthesiologist's guided intraoperative analgesia.

\section{References}

1. Gruenewald M et al. Minerva Anesthesiol. 2015; 81(5): 480-9.

Table 1. Patients' characteristics.

Age

Gender (M/F), N/N

Weight $(\mathrm{kg})$

Height $(\mathrm{cm})$

BMI

ASA physical status I/II/III, N

NSAID preoperative use, $\mathrm{N}$

Alcohol abuse, $\mathrm{N}$

Cigarette smoking, $\mathrm{N}$

Type of surgery, $\mathrm{N}$

Laminectomy

Decompression of a

narrow spinal canal

Stabilization

Fracture stabilization

Other

ASA, American Society of Anesthesiology; BMI, body mass index; M, male; F, female; SD, standard deviation; VAS, visual analog score, $\mathrm{N}$, number of patients; NSAID, non-steroid antiflammatory drug

Table 2. Perioperative data for duration of anaesthesia, sufentanil dose and desflurane concentration.

$\begin{array}{lcccc} & \begin{array}{c}\text { ANI group } \\ (\mathrm{n}=24)\end{array} & \begin{array}{c}\text { SPI group } \\ (\mathrm{n}=24)\end{array} & \begin{array}{c}\text { Control group } \\ (\mathrm{n}=24)\end{array} & \text { P-value } \\ & 120.0 & 82.0 & 94.0 & 0.230 \\ \begin{array}{l}\text { Duration of anaesthesia } \\ (\mathrm{min})\end{array} & (86.0 ; 146.0) & (73.0 ; 117.0) & (66.0 ; 125) & \\ \text { Total sufentanil dose } & 40.0 & 30.0 & 30.0 & 0.2253 \\ (\mu \mathrm{g}) & (30.0 ; 65.0) & (20.0 ; 45.0) & (20.0 ; 45.0) & \\ \text { Sufentanil dose per kg/h } & 0.236 & 0.318 & 0.256 & 0.5687 \\ (\mu \mathrm{g}) & (0.167 ; 0.386) & (0.167 ; 0.392) & (0.190 ; 0.320) & \\ \text { Desflurane }(\mathrm{vol} \%) & 4.9 \pm 0.7 & 4.6 \pm 0.5 & 4.7 \pm 0.6 & 0.230\end{array}$

Figure 1. Timing of intraoperative sufentanil boluses

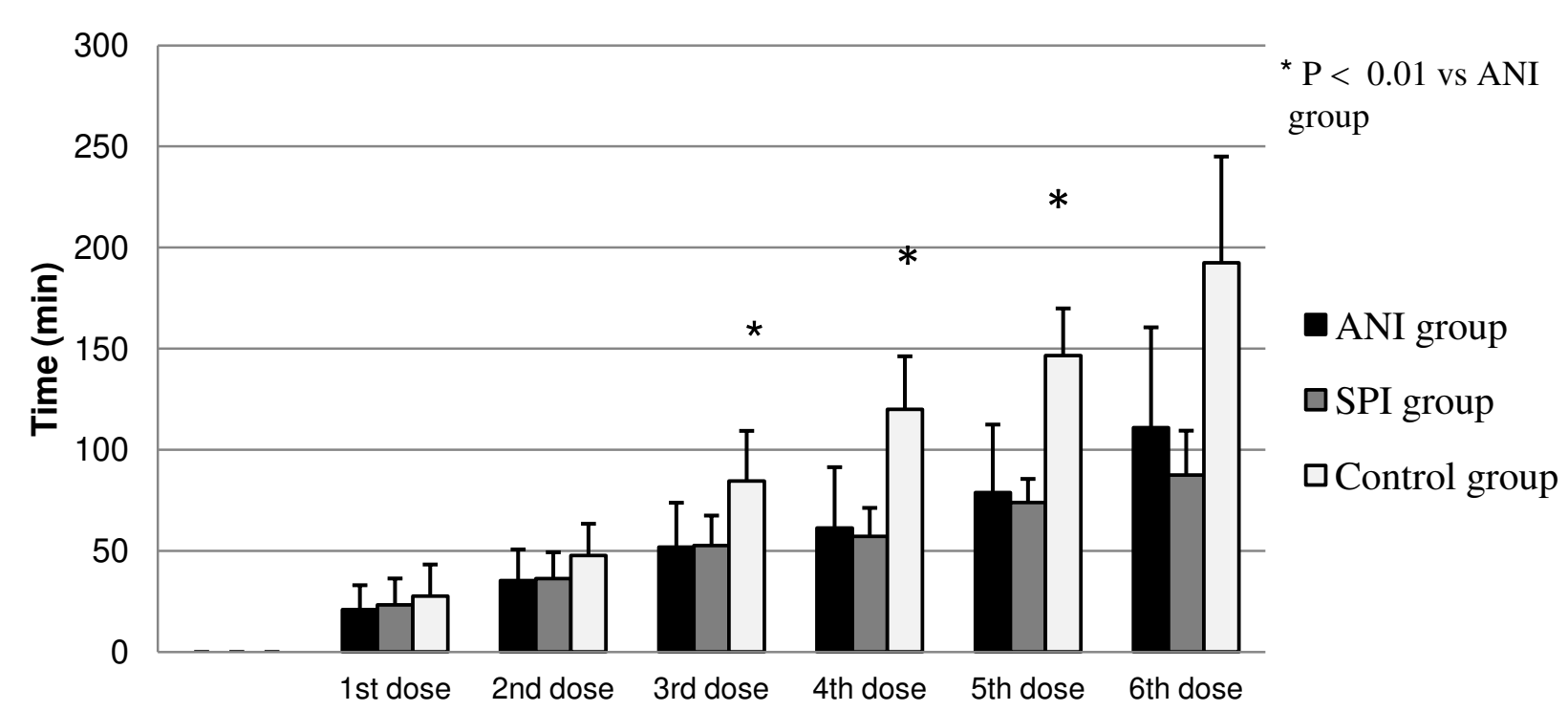

Table 3. Postoperative pain scores, serum cortisol concetrantions and postoperative complication rates

\begin{tabular}{|c|c|c|c|c|}
\hline & $\begin{array}{l}\text { ANI group } \\
(\mathrm{n}=24)\end{array}$ & $\begin{array}{l}\text { SPI group } \\
\quad(\mathrm{n}=24)\end{array}$ & $\begin{array}{c}\text { Control } \\
\text { group } \\
(\mathrm{n}=24)\end{array}$ & P-value \\
\hline $\begin{array}{l}\text { Postoperative serum } \\
\text { cortisol }(\mathrm{nmol} / \mathrm{l})\end{array}$ & $\begin{array}{c}251 \\
(124 ; 415)\end{array}$ & $\begin{array}{c}324 \\
(226 ; 564)\end{array}$ & $\begin{array}{c}268 \\
(193 ; 406)\end{array}$ & 0.4914 \\
\hline Mean VAS $1^{\text {st }}$ day & $1.06 \pm 0.57$ & $0.83 \pm 0.31$ & $1.10 \pm 0.36$ & 0.0659 \\
\hline $\begin{array}{l}\text { Length of postoperative } \\
\text { opioid use, days }\end{array}$ & $\begin{array}{c}1.00 \\
(1.00 ; 2.00)\end{array}$ & $\begin{array}{c}1.00 \\
(1.00 ; 1.00)\end{array}$ & $\begin{array}{c}2.00 \\
(1.00 ; 2.00)\end{array}$ & 0.0664 \\
\hline Postoperative & 3 & 1 & 1 & 0.4233 \\
\hline
\end{tabular}

$\begin{array}{llll}49.6 \pm 16.9 & 58.8 \pm 12.5 & 54.5 \pm 14.5 & 0.098\end{array}$

$85.8 \pm 15.5 \quad 78.0 \pm 15.5 \quad 77.7 \pm 15.5 \quad 0.133$

$\begin{array}{llll}175 \pm 11 & 170 \pm 9 & 170 \pm 10 & 0.177\end{array}$

$\begin{array}{cccc}3 / 184.3 & 26.8 \pm 4.6 & 26.7 \pm 3.9 & 0.397 \\ 4 & 2 / 19 / 3 & 1 / 20 / 3 & 0.9359\end{array}$

$\begin{array}{llll}4 & 6 & 10 & 0.226\end{array}$

$\begin{array}{llll}1 & 2 & 0 & 0.168\end{array}$

1.0000

0.5037

0.7513

0.3469

.8066 